\title{
3 Erstellung Kostensensibler Leitlinien: methodische Überlegungen aus gesundheitsökonomischer Sicht
}

\author{
Janine Biermann, Anja Neumann, Kirstin Börchers, \\ Daniela Gartner-Freyer, Petra Schnell-Inderst und Jürgen Wasem
}

\subsection{Ziele Kostensensibler Leitlinien}

Die Knappheit von Ressourcen und Begrenztheit der Mittel ist seit jeher ein zentrales Thema für die Ökonomie (vgl. etwa bereits Smith 1776). Die Ökonomie hat sich insbesondere der Frage gewidmet, wie angesichts dieser Begrenztheit der Einsatz der Mittel sinnvoll erfolgen sollte. Soweit die Ressourcenallokation nicht über den Markt erfolgt, wird thematisiert, welche nicht-marktlichen Allokationsmechanismen zu einer effizienten, den Präferenzen der Bevölkerung entsprechenden Ressourcenverwendung führen können.

Die Gesundheitsversorgung ist in den meisten Ländern überwiegend nicht marktlich organisiert. Aufgrund zahlreicher angebots- und nachfrageseitiger Besonderheiten gibt es entsprechend - um es in den Worten des seminalen Aufsatzes von Kenneth Arrow aus 1963 auszudrücken - einen „special place for medical care in economic analysis" (Arrow 1963). Diese Besonderheiten haben wesentlich zur Entwicklung von „Health Economics as a discipline“ (Culyer 1981) beigetragen .

Insbesondere die Existenz des Krankenversicherungsschutzes verhindert, dass „,normale“ Marktmechanismen greifen können. Die auf Märkten übliche Kosten-Nutzen-Abwägung des Kunden findet nämlich hier nicht statt: Die Patienten sind zwar Nachfrager nach Gesundheitsleistungen, müssen diese jedoch nicht direkt, sondern nur indirekt über den Krankenversicherungsbei- 
trag zahlen; sie sind daher eher an einer Auswahl hochwertiger als preiswerter Leistungen interessiert. Dies gilt auch für die Ärzte, die aufgrund Ausbildung und Sozialisation in Tradition des hippokratischen Eids auf das Wohl des einzelnen Patienten ausgerichtet sind und im Zweifel alle Maßnahmen, die einen Nutzenzuwachs bedeuten, unabhängig von den Kosten befürworten - was unter Wettbewerb um die Patienten verstärkt gilt. Und die Krankenversicherer müssen zwar zahlen, sind jedoch in die konkrete Auswahl der medizinischen Leistungen nicht einbezogen.

Vor diesem Hintergrund haben zahlreiche Staaten in den vergangenen Jahren quasi-staatliche Institutionen etabliert, die als ,administratives Surrogat“ für die auf Märkten von Konsumenten vorgenommene Kosten-Nutzen-Abwägung den Wert der medizinischen Interventionen aus gesellschaftlicher Sicht untersuchen und Auswahlentscheidungen für die aus öffentlichen Mitteln finanzierte Gesundheitsversorgung treffen sollen. Auch in Deutschland ist dem Gemeinsamen Bundesausschuss mit der Möglichkeit, bei fehlendem Nachweis von Nutzen und Wirtschaftlichkeit neuen Behandlungsmethoden den Zugang in die Gesetzliche Krankenversicherung zu versperren oder über Richtlinien aus der Versorgung auszuschließen oder ihren Einsatz über Therapiehinweise zu steuern, ein entsprechendes Instrumentarium zur Verfügung gestellt worden. Mit dem Institut für Qualität und Wirtschaftlichkeit im Gesundheitswesen ist dem Gemeinsamen Bundesausschuss eine wissenschaftliche Einrichtung zur Seite gestellt worden, die auf der Basis der Methoden evidenzbasierter Medizin die Datenlage aufbereiten und entsprechende Entscheidungen des Bundesausschusses vorbereiten soll.

Leistungsausschlüsse oder Therapiehinweise sind typische Beispiele „expliziter" Leistungsbegrenzungen (Marckmann 2007). Parallel dazu haben Regulierungsagenturen und Krankenversicherer in vielen Ländern Instrumente entwickelt, durch welche Restriktionen „implizit“ bewirkt werden: Hierbei ist der Leistungskatalog formal insoweit nicht beschränkt, jedoch werden die Ärzte (oder auch die Institutionen, in denen sie tätig sind, wie Krankenhäuser; auch andere Gesundheitsberufe, wie das Pflegepersonal, können betroffen sein) mit Anreizstrukturen konfrontiert, die sie anreizen (können), die Leistungen ihrerseits zu begrenzen. Beispielhaft sei ein Arzneimittelbudget genannt, das dem niedergelassenen Arzt vorgegeben ist und bei dessen Überschreitung er Gefahr einer Regresszahlung an die Krankenkasse läuft. Im Krankenhaus stellt die Vergütung über Fallpauschalen ein ähnliches Potenzial für implizite Leistungsbegrenzungen dar, auch wenn in Deutschland der Differenzierungsgrad des Fallpauschalensystems im Vergleich zum Einführungszeitpunkt stark zugenommen hat und insbesondere die Zahl der Fallpauschalen, bei denen nach dem Einsatz kostspieliger Prozeduren unterschieden wird, ausgeweitet worden ist.

Wenn auch auf die beschriebenen Formen impliziter Leistungsbegrenzungen nie komplett verzichtet werden kann, stellt sich die Frage nach der Möglich- 
keit einer Rückführung dieses Typus von Steuerungsinstrumenten insbesondere vor dem Hintergrund der damit verbundenen Konflikthaftigkeit von Begrenzungsentscheidungen für die Ärzte. Mit Kostensensiblen Leitlinien (KSLL) wird seit über 1o Jahren ein Instrument diskutiert, das eine partielle Rückführung impliziter Leistungsbegrenzungen und ihren Ersatz durch explizitere Formen der Restriktionen unterstützen könnte. Grundgedanke ist, den Kosten-Effektivitäts-Gedanken in medizinische Leitlinien zu implementieren (Mason et al. 1999): Während - so der Gedanke - medizinische Leitlinien aus klinischen Gründen abwägen, in welcher Situation den Patienten welche Maßnahme vorgeschlagen wird, soll die Kostensensible Leitlinie zusätzlich ökonomische Kriterien aus Sicht der Kostenträger oder aus gesellschaftlicher Perspektive berücksichtigen.

Entsprechend der Überlegung, dass bei begrenzten Ressourcen auch nur begrenzte Leistungen erbracht werden können (Hoppe 2009), sollen unter Berücksichtigung des Kosten-Effektivitäts-Konzepts klinische Leitlinien ökonomisch „angereichert" werden, um damit Ärzte in der Entscheidung über den Einsatz kostspieliger biomedizinischer Interventionen (insbesondere: Innovationen) bei Mittelknappheit zu entlasten.

\section{2 Überlegungen zur Methodik Kostensensibler Leitlinien}

An anderer Stelle in diesem Band wird für zwei im Rahmen des vom BMBF geförderten Forschungsprojektes „Ethische, ökonomische, rechtliche und gesellschaftliche Aspekte der Allokation kostspieliger biomedizinischer Innovationen unter finanziellen Knappheitsbedingungen " ${ }^{1}$ exemplarisch untersuchte Beispiele - die Medikamente-freisetzenden Stents (Drug Eluting Stents, DES) und die Implantation von implantierbaren Cardiovertern/Defibrillatoren (ICD) - jeweils konkret dargestellt, mit welcher Methodik die Kostensensiblen Leitlinien erstellt wurden. Ein drittes Beispiel wurde im Projektkontext entwickelt, aber nicht in diesen Band aufgenommen: Es handelt sich um eine Intervention aus dem Bereich der Intensivmedizin, nämlich den Einsatz von Drotrecogin alfa (Xigris) als adjunktive Maßnahme bei Patienten mit schwerer Sepsis. Kriterien für die Auswahl dieser Beispiele für das Forschungsprojekt waren, dass es sich zum einen um vergleichsweise teure Interventionen, zum anderem um Innovationen handeln sollte. Dies wird faktisch auch die Richtschnur für den Gemeinsamen Bundesausschuss oder andere Gremien sein müssen, wenn Kostensensible Leitlinien in den Routinebetrieb der GKV-Versorgung implementiert werden sollten.

Da die konkrete Beschreibung der Methodik in den beiden Beispielen DES und ICD an anderer Stelle in diesem Band erfolgt, soll hier demgegenüber das Vor-

1 Förderkennzeichen: 01GP0609 
3 Erstellung Kostensensibler Leitlinien:

methodische Überlegungen aus gesundheitsökonomischer Sicht

gehen allgemeiner beschrieben und über Chancen und Grenzen der Methodik reflektiert werden.

Ein erster wesentlicher Schritt bei der Entwicklung einer Kostensensiblen Leitlinie besteht darin, mittels systematischer Recherchen Evidenz einerseits bezüglich der medizinischen Effektivität und andererseits der Kosteneffektivität der zu betrachtenden Interventionen zu suchen (vgl. Schritt 1 in Abb. 1).

Hierzu ist es notwendig, mit Blick auf die medizinische Evidenz eine systematische Literaturrecherche in den einschlägigen medizinischen Datenbanken durchzuführen; die gesundheitsökonomische Entwicklung Kostensensibler Leitlinien weist insoweit diesbezüglich große Gemeinsamkeiten mit dem Vorgehen in den medizinischen Wissenschaften auf (Eccles 2001). Die gefundenen Quellen sind hinsichtlich der Relevanz für die Fragestellung zu screenen, und eine entsprechende Selektion ist zu treffen. Die Studienqualität der gefunde-

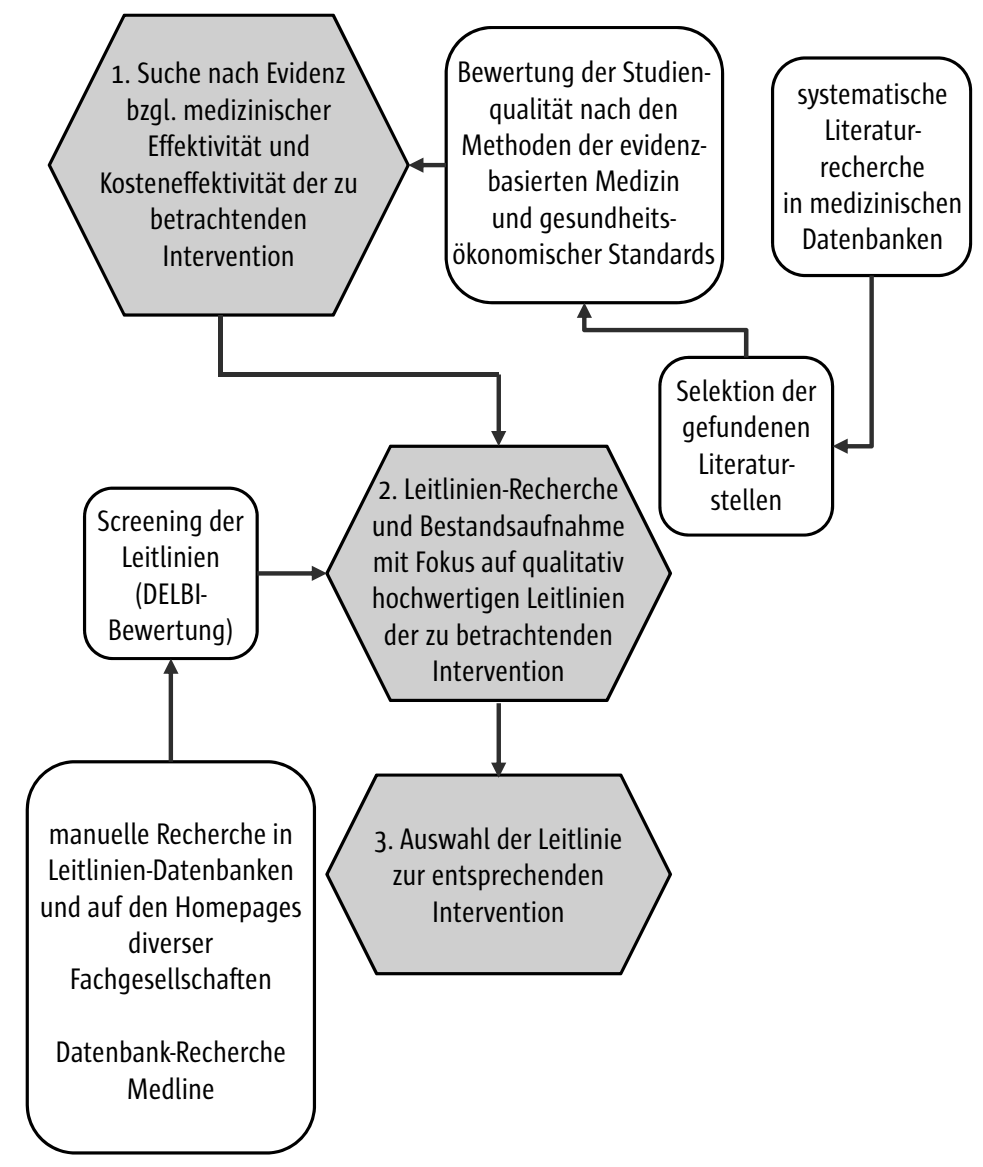

Abb. 1 Teilschritte 1 bis 3 der Erstellung einer Kostensensiblen Leitlinie (KSLL) 
nen Literatur ist zu bewerten; hierfür stehen in der Methodik der evidenzbasierten Medizin und im Bereich des Health Technology Assessment internationale wie nationale Bewertungsinstrumente zur Verfügung.

Entsprechendes gilt für die Suche nach Material für die Evidenz zur Kosteneffektivität der zu betrachtenden Intervention. Auch hier ist eine systematische Literaturrecherche durchzuführen, die die einschlägigen Datenbanken einschließt ${ }^{2}$. Bei der auch hier vorzunehmenden Bewertung der Studienqualität ist auf die gesundheitsökonomischen Standards abzustellen. Auch hierfür liegen internationale wie nationale Bewertungsinstrumente vor. ${ }^{3}$

Der nächste Haupt-Schritt besteht darin, eine Recherche auf bereits bestehende, für die zu untersuchende Fragestellung geeignete medizinische Leitlinie durchzuführen (vgl. Schritt 2 in Abb. 1). Der Crundgedanke ist hierbei, dass es nicht darum gehen kann und sollte, eine hinsichtlich der medizinischen Fragestellung neue Leitlinie zu schaffen, vielmehr soll eine bereits bestehende und akzeptierte Leitlinie hinsichtlich des gesundheitsökonomischen Kontextes „,angereichert“" werden. Sollte das Konzept der KSLL künftig in den CKVRoutinebetrieb implementiert und mit einer gewissen Verbindlichkeit ausgestattet werden ${ }^{4}$, wird zu prüfen sein, wie dieser Prozess des Generierens von gesundheitsökonomischem Inhalt in den Prozess des Erstellens der originär medizinischen Leitlinien integriert werden kann. Für den vorliegenden Fall allerdings konnte es aufgrund des straffen Zeitrahmens lediglich darum gehen, eine bereits bestehende Leitlinie $z u$ identifizieren und hinsichtlich der gesundheitsökonomischen Aspekte zu ergänzen.

Der Fokus sollte hierbei auf qualitativ hochwertigen Leitlinien bezüglich der zu betrachtenden Intervention liegen. Hierzu wurde einerseits eine systematische Datenbankrecherche, im vorliegenden Fall: in MEDLINE und EMBASE, durchgeführt. Andererseits wurden manuelle Recherchen in Leitlinien-Datenbanken durchgeführt. Auch wurde auf den Homepages diverser für die medizinischen Interventionen jeweils einschlägiger Fachgesellschaften recherchiert.

Die medizinischen Leitlinien wurden nach ihrer Identifikation einem Screening durch zwei unabhängige Reviewer unterzogen. Es erscheint für den deutschen Kontext sachgerecht, sich hierfür des DELBI-Instrumentariums zu bedienen. Dieses Deutsche Instrument zur methodischen Leitlinien-Bewertung wurde unter der Federführung der Arbeitsgemeinschaft der Wissenschaftlichen Medizinischen Fachgesellschaften e.V. (AWMF) und des Ärztlichen Zentrums für Qualität in der Medizin (ÄZQ), unter Mitwirkung auch des Medizinischen Dienstes der Spitzenverbände der Krankenkassen, erstellt (Beyer et al.

2 Dies sollte im Regelfall auch gesundheitsökonomische Datenbanken einschließen. In den in diesem Band dargestellten Anwendungsbeispielen konnte dies aus Ressourcengründen nur teilweise realisiert werden.

3 Vgl. etwa die diversen Checklisten, die im Rahmen des vom BMG Ende der goer-lahre geförderten Aufbaus einer HTA-Infrastruktur entwickelt wurden.

4 Vgl. dazu die juristischen Beiträge Kap. 7 u. 8 in diesem Band. 
3 Erstellung Kostensensibler Leitlinien:

methodische Überlegungen aus gesundheitsökonomischer Sicht

2008). Es identifiziert acht Domänen hinsichtlich derer eine Leitlinie beurteilt werden sollte: der Geltungsbereich und Zweck, die Art und Weise der Einbindung von Interessengruppen, die methodische Exaktheit der Leitlinienentwicklung, die Klarheit und Gestaltung der Leitlinie, das Ausmaß der Generalisierbarkeit ihrer Anwendung, die Frage der redaktionellen Unabhängigkeit bzw. möglicher Verflechtungen und Interessenkonflikte, ihre Anwendbarkeit im spezifischen Kontext des deutschen Gesundheitssystems und die methodologische Exaktheit der Leitlinienentwicklung bei Verwendung bereits zuvor existierender Leitlinien. Das Screening anhand dieser Domänen hat sich im vorliegenden Projektkontext als sinnvoll erweisen.

An die mit dem Screening durch eine DELBI-Bewertung abschließende Leitlinien-Recherche und -Bestandsaufnahme schließt sich schließlich die Auswahl der Leitlinie für den weiteren Prozess an (vgl. Schritt 3 in Abb. 1).

Der vierte Schritt ist das Screening der ausgewählten Leitlinie und die Darstellung der Empfehlungen dieser Leitlinie (vgl. Schritt 4 in Abb. 2). Für die Anreicherung der Leitlinie um gesundheitsökonomischen Kontext erweist sich als eine ganz zentrale Fragestellung die Durchführung von Subgruppenanalysen (vgl. Schritt 5 in Abb. 2). Die Motivation hierzu ergibt sich aus der Überlegung, dass zu vermuten ist, dass in der medizinischen Leitlinie für

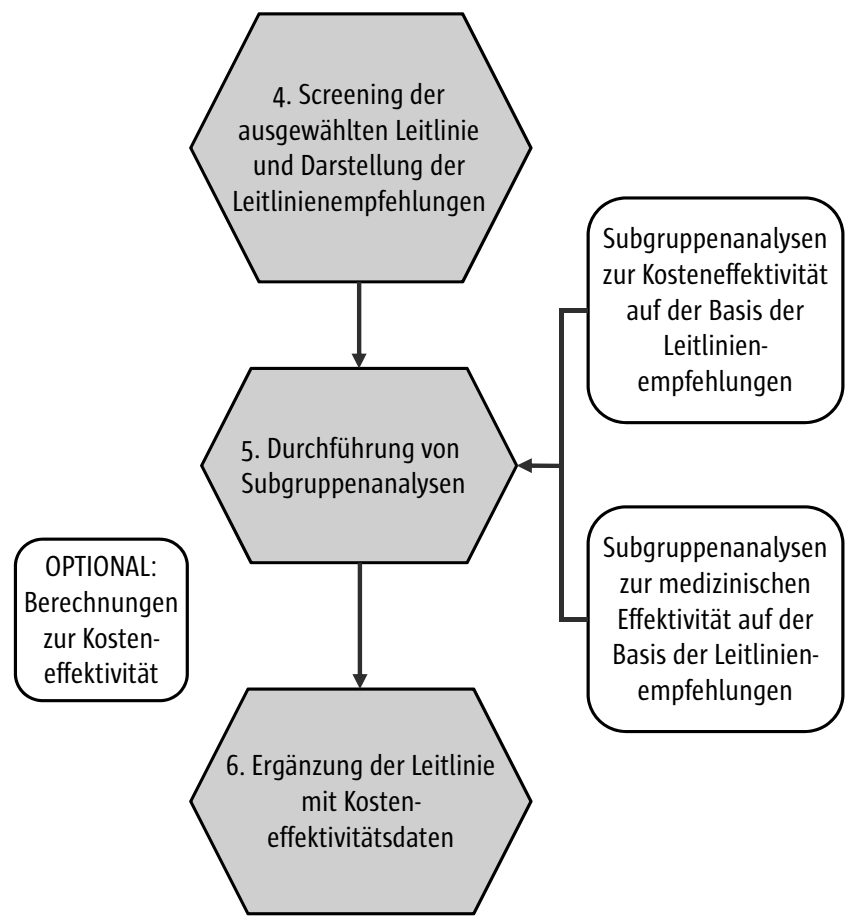

Abb. 2 Teilschritte 4 bis 6 der Erstellung einer Kostensensiblen Leitlinie (KSLL) 
sämtliche Subgruppen, für die es medizinische Evidenz für einen positiven Effekt durch den Einsatz der interessierenden Intervention gibt, eine Empfehlung zum Einsatz dieser Intervention ausgesprochen wird. Aus gesundheitsökonomischer Sicht ist jedoch nicht unwahrscheinlich, dass sich die Relation zwischen dem positiven Effekt und den hierfür aufzuwendenden Ressourcen, also die Kosteneffektivität, zwischen verschiedenen Subgruppen unterscheidet - und je nach Ausmaß des Unterschiedes in der Kosteneffektivität kann hieraus möglicherweise in einer Kostensensiblen Leitlinie die Empfehlung resultieren, für bestimmte Subgruppen (bei denen Kosteneffektivität vorliegt) die Intervention einzusetzen, bei anderen Subgruppen (bei denen Kosteneffektivität nicht vorliegt), die Intervention hingegen zu unterlassen. ${ }^{5}$

Bei dieser Recherche ist daher zweispurig vorzugehen: Einerseits muss es darum gehen, Subgruppen zur medizinischen Effektivität, andererseits zur Kosteneffektivität zu identifizieren. Auf Basis dieser Informationen gilt es dann, die originäre Leitlinie mit Kosteneffektivitätsdaten zu ergänzen (vgl. Schritt 6 in Abb. 2). Dazu ist es möglicherweise erforderlich, eigene Berechnungen zur Kosteneffektivität durchzuführen. Dies kann den Einsatz gesundheitsökonomischer Modellierungstechniken (Entscheidungsbäume, Markov-Modelle, Discrete Event Simulation u.ä.) (Wasem u. Siebert 1999) einschließen.

\subsection{Herausforderungen}

In diesem Abschnitt soll zum einen über die Limitationen berichtet werden, auf welche die Autoren bei der Erstellung der exemplarischen Kostensensiblen Leitlinien gestoßen sind. Zum anderen beziehen wir uns auf Limitationen, die sich unseres Erachtens beim Einsatz des Instrumentariums in der Versorgungspraxis ergeben.

Ein erster Block von Limitationen ergibt sich im Zusammenhang mit der Qualität der medizinischen Leitlinien. Zunächst ist festzustellen, dass interventionsspezifische Leitlinien nicht immer qualitativ hochwertig verfasst sind; hierfür fanden wir in den Anwendungskontexten des Förderprojektes eines Reihe von Beispielen. Darüber hinaus kann - worauf der DELBI-Kriterienkatalog mit Recht hinweist - nicht immer davon ausgegangen werden, dass eine hinreichende Unabhängigkeit von Politik und Industrie in den in LeitlinienGenerierungs-Kontexten im Allgemeinen unausweichlichen Konsensusverfahren besteht.

Es ist für den Einsatz von KSLL in Ressourcen-Limitations-Kontexten aber zwingend das Vorhandensein von qualitativ hochwertigen interventionsspezifischen Leitlinien zu fordern. Nach dem „Stufenschema“ der Arbeitsgemeinschaft der Wissenschaftlichen Medizinischen Fachgesellschaften e.V. er-

5 Zu den damit angesprochenen rechtlichen Fragen vgl. die juristischen Beiträge Kap. 7 u. 8 in diesem Band. 
scheint es sinnvoll, nur Leitlinien des Typs $S_{3}$ zu entwickeln, die systematisch und logisch entwickelt sind, systematisch und formal konsentiert wurden und - in der Terminologie der AWMF - eine Entscheidungs- und OutcomeAnalyse verwenden (Kopp 2010). Es ist nicht davon auszugehen, dass solche Leitlinien regelhaft zur Verfügung stehen. Sollte das Instrument der Kostensensiblen Leitlinien für den Einsatz in Ressourcen-Limitations-Kontexten erwogen werden, ist daher - auch unter Beachtung rechtlicher Aspekte - zu diskutieren, welche Abstriche gemacht werden können.

Des Weiteren ist es erforderlich, dass die notwendigen Daten zur Kosteneffektivität bei spezifischen Subgruppen von Patienten existieren. In den relevanten klinischen Studien müssen also entsprechende Subgruppen identifiziert und in der Analyse berücksichtigt worden sein. Gleiches gilt für die ökonomischen Studien zu den Ressourcenverbräuchen. Darüber hinaus müssen diese Daten auch für die Auswertung zur Verfügung stehen - möglicherweise wird auf die Durchführung von Subgruppenanalysen in den einschlägigen Publikationen zwar hingewiesen, die Daten sind jedoch nicht in notwendigem Umfang publiziert: In diesem Fall kann versucht werden, mit den Autoren der Originalstudien Kontakt aufzunehmen, was jedoch (so die Erfahrung in dem vorliegenden und in weiteren Projekten, an denen die Verfasser beteiligt waren) in vielen Fällen nicht erfolgreich zum Abschluss gebracht werden kann.

Studien, aus denen die Daten für die Leitlinie und auch für die KSLL generiert werden, sind oft multinational angelegt. Damit stellt sich die Frage nach der Vergleichbarkeit über Ländergrenzen hinweg und für das deutsche Gesundheitssystem. Diese Fragestellung ist in den vergangenen Jahren intensiv diskutiert worden, und die Herausforderungen einer Übertragbarkeit sind herausgearbeitet worden (Welte u. Leidl 1999, Welte et al. 2004). Es kann jedenfalls im Regelfall nicht unreflektiert davon ausgegangen werden, dass die Daten zur Kosteneffektivität ohne Weiteres auf Deutschland übertragen werden können. In vielen Fällen wird es vielmehr so sein, dass Adaptionen vorzunehmen, das heißt: eigenständige Berechnungen durchzuführen sein werden.

Sofern in internationalen Kosteneffektivitätsstudien über die Ermittlung und Präsentation der Daten hinaus auch wertende Aussagen zum Vorliegen oder Nicht-Vorliegen von Kosteneffektivität gemacht werden, können diese Aussagen nicht auf den deutschen Kontext übertragen werden, da die nationalen Zahlungsbereitschaften für Zusatznutzen medizinischer Interventionen unterschiedlich sind. Vor dem Einsatz von KSLL im deutschen Kontext bedürfte es vielmehr einer wertenden Entscheidung der hierfür zuständigen Institutionen - vermutlich wäre dies der Gemeinsame Bundesausschuss - zur Frage, wann noch Kosteneffektivität vorliegt und bei welcher inkrementellen Kosteneffektivitäts-Relation sie nicht mehr gegeben ist.

Eine weitere potenzielle Limitation ergibt sich aus der Notwendigkeit, eine aktuelle Leitlinie zu verwenden, wenn der Einsatz von KSLL zur Begrenzung von Ressourcen im medizinischen Handeln vertretbar sein soll. Nicht immer 
sind die Leitlinien hinreichend aktualisiert. Außerdem gilt die gleiche Anforderung für die KSLL selber: Eine einmal entwickelte KSLL wird regelmäßig zu aktualisieren sein - dies bezieht sich zum einen auf den medizinischen Sachverhalt, gilt aber auch für die ökonomische Seite. Es ist nämlich davon auszugehen, dass (zumindest wenn die Perspektive der Kostenträger, also der Krankenkassen, eingenommen wird) die Kostenseite dynamischen Veränderungen unterliegt - beispielhaft, wenn kostenintensive Arzneimittel, die einen relevanten Teil der Gesamtkosten ausgemacht haben, generisch verfügbar werden, oder wenn ein neues Vergütungssystem für Ärzte oder Krankenhäuser eingeführt wird.

Schließlich ergeben sich Limitationen daraus, dass das Instrument der KSLL allgemein konzipiert ist, der Arzt aber konkrete einzelne Patienten zu behandeln hat. Dies kann - auch jenseits der ohnehin vermutlich immer einzuräumenden Option, Sonderfälle von der Anwendung der Kostensensiblen Leitlinie auszunehmen - auch für die „Routinefälle“ ein Problem sein - auch wenn von Ärzten vielfach das Vorhandensein von KSLL oder ähnlichen Instrumenten eingefordert wird, um Entlastung bei den Ressourcenallokationsprozessen zu bekommen. ${ }^{6}$

\subsection{Fazit}

Insgesamt kommen wir anhand der drei im Projekt explorierten Anwendungsbeispiele für die Entwicklung Kostensensibler Leitlinien, von denen wir zwei (DES und ICD) in weiteren Kapiteln dieses Buches beschreiben, zu einem gemischten Fazit: Das Erstellen von KSLL erscheint grundsätzlich durchaus leistbar. Die Realisierbarkeit ist jedoch in hohem Maße abhängig von der Qualität der vorhandenen medizinischen Leitlinien sowie der publizierten Daten zur Kosteneffektivität bzw. den Kosteninformationen.

Wie für das Erstellen von Leitlinien generell, aber auch für die Durchführung systematischer Nutzen- und Kosten-Nutzen-Bewertungen, ist von einem hohen Arbeitsaufwand auszugehen. Der Einsatz von KSLL sollte daher, wenn er denn für juristisch zulässig und politisch durchsetzbar gehalten wird, auf solche medizinische Interventionen beschränkt werden, bei denen ex ante vermutet werden kann, dass durch die ökonomische Ergänzung der Leitlinien ein hinreichendes Einsparpotenzial realisiert werden kann. Ggfs. empfiehlt sich in der Umsetzung in den Routinebetrieb ein zweistufiges Verfahren einer Schnellbewertung, der dann eine umfassendere systematische Analyse folgt. Die beiden in diesem Band an anderer Stelle vorgestellten Beispiele bargen in der ex ante-Einschätzung das Potenzial für Kosteneinsparungen; inwieweit sie durch Kostensensible Leitlinien würden gehoben werden können, zeigen die Falldarstellungen in diesem Band.

6 Vgl. dazu Kap. 9 zu den Einschätzungen von Ärzten 
3 Erstellung Kostensensibler Leitlinien:

methodische Überlegungen aus gesundheitsökonomischer Sicht

\section{Literatur}

Arrow, K.J. (1963): Uncertainty and the welfare economics of medical care. American Economic Review. 53, 5. 941-973.

Beyer, M., Geraedts, M., Gerlach, F.M., Gülich, M., Kopp, I., Lelgemann, M., Ollenschläger, G., Selbmann, H.-K., Thole, H. und Windeler, I. (2008): Deutsches Instrument zur methodischen Leitlinien-Bewertung (DELBI) Fassung 2005/6 + Domäne 8. Arbeitsgemeinschaft der Wissenschaftlichen Medizinischen Fachgesellschaften e.V. (AWMF) und Ärztliches Zentrum für Qualität in der Medizin (ÄZQ). URL: http://www.awmf.org/ fileadmin/user_upload/Leitlinien/Werkzeuge/delbi-fassung-2005-2006-domaene-8-2008-1.pdf (Zugriff am 21.04.2015)

Culyer, A. (1981): Health, Economics and Health Economics. In: J. Van der Gaag und M. Perlman (Hrsg.): Health, Economics, and Health Economics. North-Holland. Leiden. 31-51

Eccles, M. (2001): How to develop cost-conscious guidelines. York.

Hoppe, J. (2009): Verteilungsgerechtigkeit durch Priorisierung - Patientenwohl in Zeiten der Mangelverwaltung. URL: http://www.bundesaerztekammer.de/page.asp?his=0.2.6499.7209 (Zugriff am 21.04.2015)

Kopp, I. (2010): Perspektiven der Leitlinienentwicklung und -implementation aus Sicht der AWMF. Z. Rheumatol. 69, 298-304. DOI 10.1007/s00393-009-0526-3

Marckmann, G. (2007): Zwischen Skylla und Charybdis: Reformoptionen im Gesundheitswesen aus ethischer Perspektive. Gesundheit ökonom Qual manag. 12, 96-100.

Mason, J., Eccles, M., Freemantle, N. und Drummond, M. (1999): A framework for incorporating cost-effectiveness in evidence-based clinical practice guidelines. Health Policy. 47, 37-52.

Smith, A. (1776): An Inquiry into the Nature and Causes of the Wealth of nations. W. Stratman. London.

Wasem, J. und Siebert, U. (1999): Gesundheitsökonomische Parameter einer Evidence-based medicine. Zeitschrift für ärztliche Fortbildung und Qualitätssicherung. 93, 427-436.

Welte, R., Feenstra, T., Jager, H. und Leidl, R. (2004): A decision chart for assessing and improving the transferability of economic evaluation results between countries. Pharmacoeconomics. 22, 13. 857-76. 22134 [pii]

Welte, R. und Leidl, R. (1999): Übertragung der Ergebnisse ökonomischer Evaluationsstudien aus dem Ausland auf Deutschland. In: R. Leidl, J.M. von der Schulenburg und J. Wasem (Hrsg.): Ansätze und Methoden der ökonomischen Evaluation - eine internationale Perspektive. Baden-Baden. 171-202 